\title{
THE SWELLING PRESSURES OF NORMAL AND GLAUCOMATOUS VITREOUS BODIES
}

\author{
BY
}

\author{
Sir Stewart Duke-Elder, Hugh Davson \\ and GRAHAM HARVEY BENHAM
}

FROM THE DEPARTMENT OF PHYSIOLOGY AND BIOCHEMISTRY,

UNIVERSITY COLLEGE, LONDON

IN earlier studies (Duke-Elder et al., 1935; Duke-Elder and Davson, 1935) the possibility was discussed that a swelling of the vitreous body is the cause of primary chronic glaucoma. The results obtained pointed against the theory, but till now a decisive proof has been lacking. In the present work a technique for measuring the swelling pressure of the vitreous body has been developed and applied to normal and glaucomatous vitreous bodies. If a swelling of the vitreous body is responsible for the raised intraocular pressure in primary chronic glaucoma, then it would seem to be necessary that its swelling pressure in this disease must be greater than normal. The present investigation was undertaken primarily with a view to settle this point.

The observation by Duke-Elder (1930) that the isolated vitreous proteins only swell to about forty times their weight in water, in conjunction with certain other facts led us to believe that the vitreous body has been formed as a clot from a sol (Duke-Elder and Davson, 1935) so that dehydration by pressure was considered as an essentially irreversible phenomenon producing states of instability; it was thus considered as incorrect to speak of a true swelling pressure in this case. Baurmann (1934), however, has shown that if the vitreous proteins are not allowed to dry during their preparation they may be made to swell back to a dilution of the same order as that in the original vitreous body from which they were derived. It thus seems that the vitreous body is formed by a process of swelling and not as a dilute clot from a sol and that a genuine swelling pressure must exist however small at all states removed from the swelling maximum.

In considering the rôle of the swelling pressure of the vitreous body in glaucoma from the theoretical point of view, it would not be necessary for the abnormal swelling pressure of the gel to be equal to the difference in pressure between the normal and the glaucomatous eve. The exact amount of the increase which would be necessary is difficult to determine for any individual case, as it might depend on the vascular changes accompanying the raised intra-ocular pressure. Thus if the vitreous body were to exhibit a 
very slightly raised swelling pressure it would absorb water and swell, thereby increasing the intra-ocular pressure. However, the capillary pressure would of necessity rise in response to the raised intra-ocular pressure in a purely mechanical way (Duke-Elder, 1926), so that the condition intra-ocular pressure=capillary pressure-Donnan osmotic difference would be satisfied and equilibrium would be established at a new level. The vitreous body would therefore be able to attract more water, and as the rise in intraocular pressure produced in this way would also be accompanied by a rise in the vascular pressures, one can conceive a gradual mounting up of the pressure far greater than the swelling pressure of the vitreous body in its postulated abnormal state. The problem therefore resolves itself into placing a lower limit on the swelling pressure required to produce a final state of tension of (say) $60 \mathrm{~mm} . \mathrm{Hg}$. If the swelling of the vitreous body is to cause a raised intra-ocular pressure, the extra water held by it must come from the capillaries, since only in this way will the total volume of the eye-contents be increased; although if the force with which the vitreous body attracts this water is very small, i.e., if the swelling pressure is very small, the vitreous body will satisfy its demands for water at the expense of the fluid in the eye rather than from the capillaries since in the former case no work against an external pressure will have to be done. It is well known that in a number of cases of absolute glaucoma fluid in considerable quantities is found in the vitreous cavity; in fact this excess of fluid seems to push forward the lens, diminish the anterior chamber, compress the ciliary body, choroid and retina, and to be the ultimate cause of the raised pressure of the eye. If this accumulation of fluid and its retention is due to its being held by the vitreous gel owing to an increase of its swelling pressure, then, in view of the theoretical arguments put forward in this paragraph, we may say for the purposes of argument, that the swelling pressure of the vitreous body in glaucoma of this type must be at least of the order of $1 \mathrm{~mm}$. Hg. If it can be shown to be greater than this, the theory that an abnormally increased swelling pressure in the gel is responsible for primary chronic glaucoma receives more support. If it is shown that the swelling pressure is less than this, then it would appear to be advisable to seek in some other physico-chemical mechanism an explanation of the pathological phenomena we are investigating. If, on the other hand, there appears to be no difference in swelling pressure between the normal and abnormal vitreous gel, and especially if both values are small, the hypothesis must be rejected.

The results of the measurements described in this paper show that the swelling pressure of a glaucomatous human vitreous body is not sufficient to account for the raised tension. 


\section{Experimental}

The principle of the apparatus is illustrated in Fig. 1. Chamber A contains a sintered glass plate, $\mathrm{B}$, and is connected to a graduated tube $\mathrm{C}$ by rubber tubing. The apparatus is filled with Ringer solution and by manipulation of $T_{1}$ and $C$ the latter is adjusted so that the fluid just wets the plate $B$ while the meniscus and the plate are on the same level. The vitreous body is placed in A after

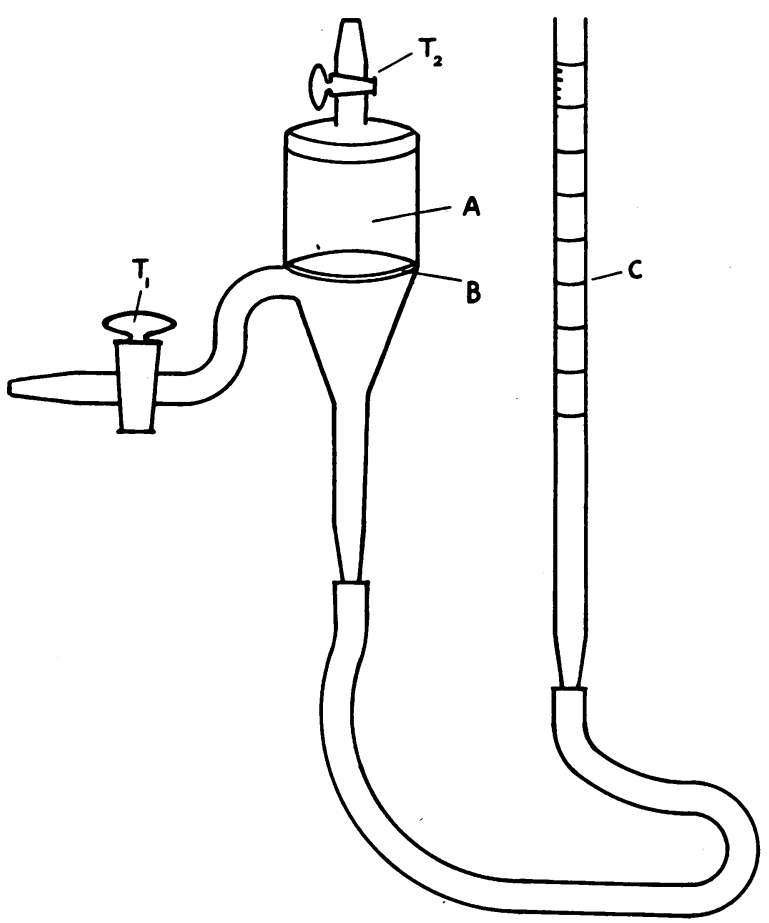

Fig. 1.

closing $T_{1}$ and $C$ is raised so that the meniscus in $C$ is at the same level as the top of the vitreous body. $T_{2}$ is kept open during the whole of the experiment. By lowering $\mathrm{C}$ any required pressure may be exerted on the vitreous body, so that water is withdrawn from it. The meniscus rises into $C$ until the difference in level between the meniscus in $C$ and the top of the vitreous body in $A$ represents the swelling pressure of the vitreous body at the given state of dehydration. Thus if the tube is lowered by $2 \mathrm{~cm}$. and the meniscus rises so that the difference in level is $1 \mathrm{~mm}$., the swelling pressure may be said to be not greater than $1 \mathrm{~mm}$. saline over the volume change corresponding to the loss of water produced in 
this way. The volume change is measured by observing the changes in height of the meniscus in the graduated tube and multiplying by the cross-sectional area. To obtain an exact value of the swelling pressure would take a long time as a film of protein is formed on the porous plate through which water filters only very slowly; however, as the purpose of these experiments is to investigate whether a swelling pressure of at least 1 or $2 \mathrm{~mm}$. $\mathrm{Hg}$ exists, it is sufficient to note the position of the meniscus after a short time and regard the pressure registered as an upper limit.

Normal, dialysed and KCNS-treated ox vitreous bodies and a human glaucomatous vitreous body were used; in the last case the vitreous body was taken from a freshly enucleated eye in absolute glaucoma with high tension.*

\section{Results}

With all the vitreous bodies examined identical results were obtained; the upper limit of the swelling pressure at volumes of 100 to $<1$ being within the experimental error (1-2 mm. saline). To illustrate the method the protocol from a normal vitreous body is shown.

\begin{tabular}{ccccccc} 
Volume of vitreous body & Time & \multicolumn{2}{r}{ Swelling pressure } \\
$100-59$ & $\ldots$ & 1 hour & $\ldots$ & $>7 \mathrm{~mm}$. saline \\
$59-57$ & $\ldots$ & 2 hours & $\ldots$ & $>4$ &, &, \\
$57-56$ & $\ldots$ & $3, n$ & $\ldots$ & $>2$ &, &, \\
$56-16$ & $\ldots$ & $24,$, & $\ldots$ & $>1$ &, &, \\
$16-<1$ & $\ldots$ & $30,$, & $\ldots$ & $>3$ &, &,
\end{tabular}

From this protocol it is seen that at the volumes 56 to 16 the pressure was $>1-2 \mathrm{~mm}$. saline (the limits of experimental errors); from this it may be inferred that at all volumes greater than 16 the swelling pressure must be less than this value, since the swelling pressure of a gel decreases with increasing volume. This was confirmed in other experiments in which attention was concentrated on the earlier phases of dehydration in so far as it was shown that the swelling pressures here also fell within the limits of experimental error. From the same protocol it appears that at a volume of $<1$ the swelling pressure is $>3 \mathrm{~mm}$. saline: from other experiments where attention was concentrated on this phase the limit could be brought down to $1-2 \mathrm{~mm}$. saline as with greater volumes. By a volume of $<1$ is meant the volume of the vitreous body when it appears as a hydrated skin on the porous plate with a volume of perhaps $0.05 \mathrm{ml}$. In this region the volume of gel

*We are indebted to Dr. Briggs, of Moorfields Hospital, for providing us with this eye 
remaining is too small to allow of the measurement of any further changes in hydration with pressure with the experimental arrangement used, so that all experiments had to stop when this state was reached. As will be shown in this discussion, it is probable that the swelling pressure reaches considerable magnitudes in this region. However the aim of the experiments described here are primarily to determine the swelling pressures at volumes only slightly removed from normal as, in the case of a glaucomatous vitreous body, it is the pressure in this region which determines the possibility of its swelling in vivo against an externally imposed force.

\section{Discussion}

The first conclusion to be drawn from the results is that the swelling pressure of the vitreous body cannot be an aetiological factor in primary glaucoma. The most surprising feature of the results is the failure of the vitreous body to exhibit a swelling pressure of $1-2 \mathrm{~mm}$. saline or more even when dehydrated by over 99 per cent.; further, it might at first sight have been expected that a strong hydrating agent like KCNS would have produced a swelling pressure of this order or greater in this range of volumes, but such was not the case; the result is in general agreement with earlier findings oro the elasticity (Duke-Elder and Davson, 1935). The results allow of some interesting conclusions in regard to the nature of the vitreous body. Thus, in the first place, since the vitreous body ha very pronounced elastic properties and contains only 0.025 per cent. of the gel-forming protein (Duke-Elder, 1930), it must be concluded that the protein micelles are linked together in branching chains, a conclusion already suggested by another physico-chemical line of approach (Duke-Elder and Robinson, 1934). With a gel of such low protein content there can be no possibility that the bulk of water is held by hydration forces, i.e., electrostatic attraction of water dipoles, as the following considerations will show. From the titration curve of the vitreous protein (Duke-Elder, 1930), it appears that at normal $\mathrm{pH}$ about $60 \times 10^{-5}$ equivalents of base are bound by one gramme of protein. With a normal vitreous body this would mean $1.5 \times 10^{-7}$ equivalents per ml. and roughly corresponds to the number of - $\mathrm{COO}-$ ions per ml. of vitreous body. Assuming for simplicity that they are distributed in a cubic lattice, their distance apart, $l$, will be given by substitution in the formula:

$$
l=\sqrt{\frac{1}{\mathrm{NC}}}
$$

where $\mathrm{N}$ is Avogradro's number and $\mathrm{C}$ the concentration of - $\mathrm{COO}-$ ions in equivalents per $\mathrm{ml}$. By substitution in this way a value for $l$ equal to $2 \times 10^{-6} \mathrm{~cm}$. or 200 Angström units is obtained. 
Born (1927) has shown that the radius of the $\mathrm{Li}+$ ion plus its hydration sheath is $2 \cdot 26 \AA$, whilst the radius of the ion itself is $0.45 \AA$; the forces of hydration, in this case operate over a distance $1.81 \AA$, As the $\mathrm{Li}+$ ion is far more heavily hydrated than the - $\mathrm{COO}$ - ion, and further, as Hartley (1936) has pointed out, as there is no reason to suppose that the $-\mathrm{COO}^{-}$in a protein micelle

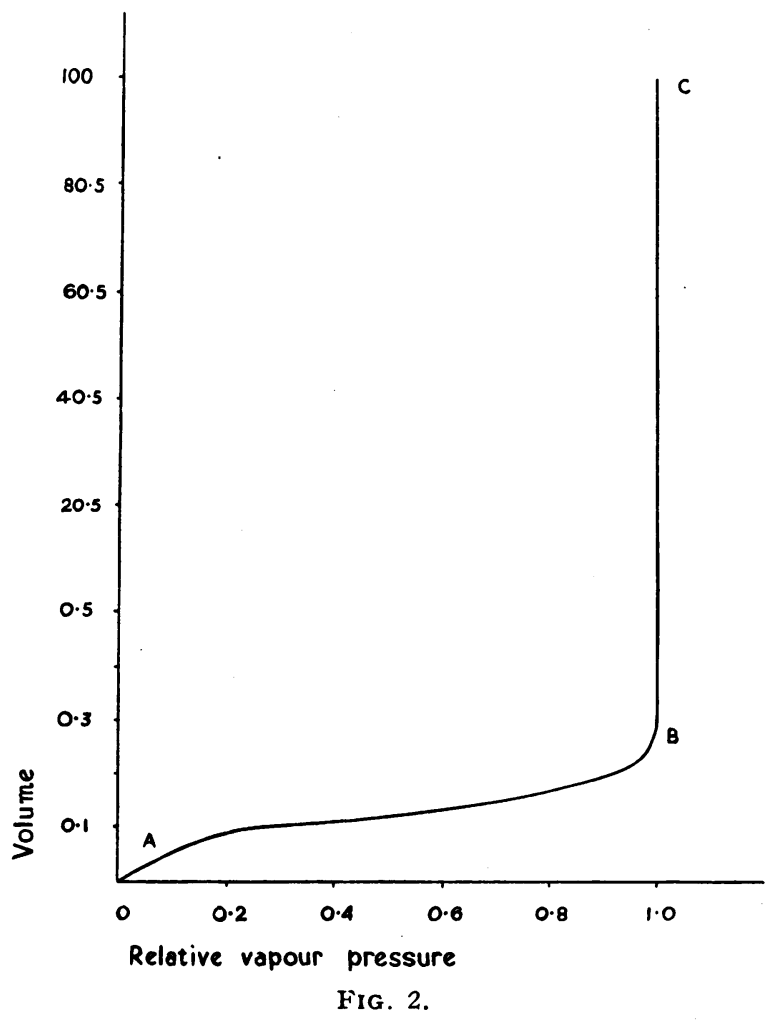

will have a very different hydration from that of the $-\mathrm{COO}-$ ion in $\mathrm{Na}$ acetate, it is evident that it is quite out of the question to consider the existence of any pull on the water molecules at distances of $100 \AA$.

These considerations are confirmed by the vapour pressure isotherm of the vitreous body. In Fig 2 a curve showing the vapour pressures of the vitreous body at different water contents is shown. The figures used for plotting this curve are taken from Duke-Elder (1930) and are corrected approximately for the salt content of the vitreous body. In order to bring out the typical $\mathrm{S}$-shape of the curve at low volumes, the ordinates above a volume 
of $0.5 \mathrm{ml}$. are one hundred times those below this value. Thus if the curve were drawn with the ordinates equal all the way up the vertical distance between $B$ and $C$ would be approximately one hundred times that between $B$ and $A$. The vapour pressure of the vitreous body only begins to decrease at a volume of 0.3 per cent. of the original volume, so that it is at this point that any appreciable diminution of the activity of the water molecules occurs. At the point $\mathrm{B}$, then, there is a sharp transition point between water held by electrostatic and co-ordinate valency forces and water not so held.

Thus there is a duality in the nature of the water held by the vitreous body which is reflected in its numerous macro- and microscopical peculiarities. In this region characterized by the part $A B$ of the graph the vitreous protein has a typical lyophilic attraction for water and should have a swelling pressure of the same order as that of concentrated gelatin gels. In the region characterized by $\mathrm{BC}$ there is no actual attraction for water whilst the amount of water associated with the protein is very great; it is in this region, therefore, that the vitreous body would be expected to show characteristics different from those of the conventionally studied gels. Since the water in this region is not held by hydration forces, one can only conclude that the vitreous protein is oriented in a framework of pores which contain this water and so give the gel a certain degree of solidity. Whether these pores are formed by parallel fibres of protein or by a series of spiral chains is not easy to decide; the similarity of the vitreous protein to the sclero proteins might lead one to prefer the latter possibility (Astbury, 1933). It is probable that it is this duality in the nature of the water contained in the vitreous body, with the huge preponderance of one form, which has led some authors to think that the vitreous body is fundamentally different from other gels, in fact, to deny that it is a gel at all. However, a consideration of the parallel processes of swelling of the vitreous protein and of gelatin (say) in water, shows that the difference is by no means so fundamental as to preclude the vitreous body from the category of gels. Thus when the former swells there is an initial strong hydration of the protein micelles whereby the chains are separated and oriented to form pores. The hydration, by inducing ionisation of the $-\mathrm{COOH}$ groups, produces net negative charges at localised points in the chains; these negative charges repel each other, thus enlarging the pores and making room for more and more water. With gelatin the same process of hydration and pore formation occurs but the forces of hydration are strong enough to rupture the micellar linkages before the pores expand to anything like the extent they do in the vitreous body, so that the gel passes into a sol. Thus the essential difference between the gel-forming powers of gelatin and the vitreous protein resides in the power of the micellar units of 
the latter to adhere to each other and resist the hydration forces which tend to rupture them.

It may be objected that in explaining the nature in which the water is associated with the vitreous body we have rejected the idea that an electrostatic attraction for water dipoles may occur over large ranges, whilst retaining the notion that an electrostatic repulsion between two negative charges may operate over comparable distances. This objection is by no means valid, however, as the attraction of a central ion for a dipole depends on a number of factors including the divergence of the field round the central ion and the thermal energy of the dipole, so that the effectiveness of the attractive force falls off far more rapidly with distance than in the case of the repulsion of two like non-dipolar charges.

\section{Conclusions}

1. The swelling pressure of the normal vitreous body is very small-less than 1 to $2 \mathrm{~mm}$. saline.

2. This value is not materially increased by dialysation or in the presence of a strong hydrating agent.

3. The swelling pressure of the vitreous gel in chronic primary glaucoma is of the same order.

It therefore follows that the swelling pressure of the vitreous body cannot be the cause of chronic primary glaucoma. It is proposed in a forthcoming publication to produce experimental evidence in favour of a theory implicating a different physicochemical mechanism in the causation of this disease. Some theoretical deductions are made from these experiments as to the structure of the vitreous gel and the means by which it holds its water.

We gratefully acknowledge our indebtedness to Professor Freundlich and Mr. Hartley, of the Chemistry Department, for advice and criticism and to the Medical Research Council for personal grants to two of us (H. D. and G. H. B.) and for defraying the whole expenses of this work.

\section{REFERENCES}

Astbury.-Fundamentals of fibre structure. London, 1933.

Baurmann.-Arch.f. Ophthal., Vol. CXXXII, p. 302, 1934.

Born.-Z. Electrochem., Vol. XXVI, p. 401, 1927.

Duke-Elder.-Jl. of Physiol., Vol. LXI, p. 409, 1926; Brit.Jl. of Ophthal., Vol. X, p. 513, 19:6.

Brit. Jl. of Ophthal., Monograph, Supplement IV, 1930.

Duke-Elder and Davson.-Biochem. Jl., Vol. XXIV, p. 1121, 1935 : Brit. Jl. of Ophthal., Vol. XIX, p. 433, 1935.

Duke-Elder and Robertson.-Brit. Jl. of Ophthal., Vol. XVIII, p. 433, 1934.

Duke-Elder, Davson and Robertson.-Biochem. Jl., Vol. XXIX, p. 72, 1935.

Hartley.-Aqueous solutions of paraffin chain salts, p. 59. Paris, 1936. 